\title{
基于分子自旋阀的自旋电子学
}

\author{
谷现荣 ${ }^{1,2}$, 郭立丹 ${ }^{1,3}$, 秦阳 ${ }^{1,2}$, 孙向南 ${ }^{1,2 *}$
}

1. 国家纳米科学中心, 中国科学院纳米系统与多级次制造重点实验室, 中国科学院纳米科学卓越创新中心, 北京 100190 ;

2. 中国科学院大学纳米科学技术学院, 北京 100049 ;

3. 中国石油大学(北京)新能源与材料学院, 材料科学与工程系, 北京 102249

* 联系人, E-mail: sunxn@nanoctr.cn

2018-08-14 收稿, 2018-09-17 修回, 2018-09-18 接受, 2018-10-24 网络版发表

国家自然科学基金(21673059)、国家重点研发计划“纳米科技”重点专项(2017YFA0206600, 2016YFA0200700)、中国科学院科研仪器设备研 制项目(YJKYYQ20170037)和中国科学院率先行动“百人计划”资助

\begin{abstract}
摘要＼cjkstart分子半导体材料因具有很长的自旋驰豫时间, 被认为在自旋电子学领域存在巨大的应用潜力. 在基于分 子半导体材料开展的自旋电子学研究被首次报道后的十余年里, 以分子自旋阀为载体的自旋电子学研究取得了巨 大发展并引起了广泛关注. 本文将围绕分子自旋阀中的自旋注入、界面效应和输运等关键研究方向, 综述近年来 该研究领域的重要研究成果, 具体包括: 分子自旋阀制备工艺改善、结构优化对自旋注入效率的提升, 自旋界面 效应对优化注入和调控信号等方面的最新进展; 以及分子半导体中自旋输运距离优化和输运机制研究结果. 最 后, 基于分子自旋沎中的注入、界面效应和输运的研究基础, 展望分子自旋㑈多功能化这一新兴研究方向的发展 前景. 以上进展对未来自旋电子学和分子电子学领域进一步交叉研究的开展具有借鉴价值.
\end{abstract}

关键词分子自旋阀, 分子半导体材料, 自旋注入, 自旋界面效应, 自旋输运

分子半导体材料因其独特的光、电性能, 以及在 轻质量、低成本和高柔性方面的众多优势被认为极具 应用前景, 因此以分子半导体材料及器件为核心的 分子电子学研究在过去几十年里获得了高速发 展 $^{[1 \sim 3]}$. 另外, 分子半导体材料在化学结构上主要是 由碳、氢、氧、氮等轻质元素组成, 其自旋-轨道耦 合作用很弱, 因而自旋电子在分子半导体材料中的 弛豫时间很长, 在实现高效、长距离自旋输运方面潜 力巨大 ${ }^{[4]} .2002$ 年, Dediu等人 ${ }^{[5]}$ 首次将自旋电子注人 分子半导体材料六噻吩 $\left(\mathrm{T}_{6}\right)$ 并观察到了自旋扩散信 号, 分子自旋电子学由此诞生. 这一新兴学科的研究 结合了自旋电子学 ${ }^{[6,7]}$ 和分子电子学的研究基础, 并 涉及了物理、化学、材料等多学科的深度交叉. 分子 自旋阀 (MSV) 器件研究是分子自旋电子学领域发展
的重要器件基础, 在过去的十余年里引起了世界范 围的广泛关注并取得了许多重要进展. 分子自旋阀 器件中自旋注人效率提升和界面效应研究, 以及自 旋输运距离优化和机制探索为该领域更系统的理论 研究奠定了坚实的基础. 长远来看, 基于分子自旋器 件的自旋注人、界面效应以及输运研究, 自旋阀功能 化探索必将在未来推动分子自旋电子学走向应用.

\section{1 分子自旋阀器件}

2004年，首个垂直结构的MSV制备成功，并且 在低温下实现了高达 $40 \%$ 的巨磁电阻 $(\mathrm{GMR})^{[8]}$, 自此, MSV作为分子自旋电子学研究开展的重要载体受到 广泛关注. MSV是由 2 个铁磁电极和 1 个非磁性分子 层组成的垂直的三明治结构器件 ${ }^{[9]}$. 在工作状态下, 
自旋极化载流子由一端铁磁电极注人分子层, 在其 中输运并维持自旋极化, 最终由另一端铁磁电极自 旋检出 (图1(a)). MSV器件的2个铁磁电极因矫顽力不 同, 其相对磁化方向在磁场作用下会表现出平行和 反平行两种状态, 两种状态时器件的电阻值分别标 记为 $R_{\mathrm{p}}$ 和 $R_{\mathrm{ap}}$, 而两者差值百分比称为磁电阻 $(\mathrm{MR})$, 是自旋阀器件的重要参数之一 $\left(\mathrm{MR}=\left(R_{\mathrm{ap}}-R_{\mathrm{p}}\right) / R_{\mathrm{p}}\right)$ (图 $1(\mathrm{~b}))^{[10]}$. MSV器件性能的提升可通过增强自旋注人 效率, 延长自旋输运距离的方式实现 ${ }^{[11]}$, 这些自旋 进程与器件制备工艺、器件结构优化关系密切.

\section{2 基于分子自旋阀的自旋注入优化}

自旋注人是MSV中自旋进程的起始, 是分子材

(a)

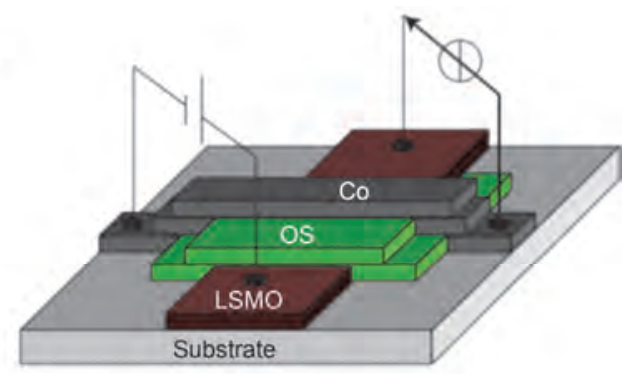

(b)

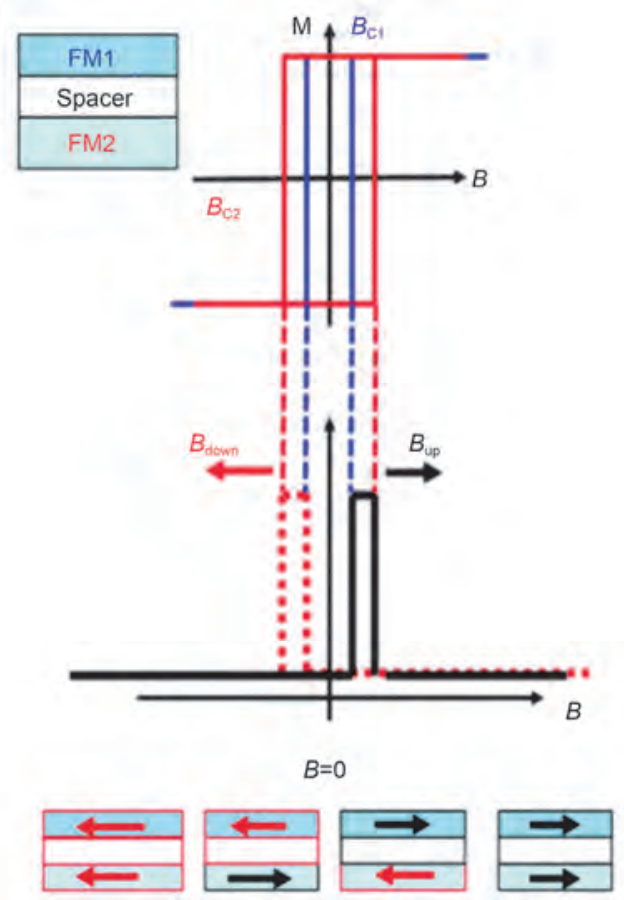

图 1 分子自旋阀的结构和表征参数. (a) MSV器件结构示意图 ${ }^{[9]}$; (b) MR原理图 ${ }^{[10]}$

Figure 1 Structure and characterization parameter of MSV. (a) Schematic structure of $\mathrm{MSV}^{[9]}$; (b) explanation of $\mathrm{MR}^{[10]}$
料中自旋输运实现的必要前提, 也是决定MSV器件 性能的关键因素. 然而, 由于MSV垂直结构的限制 以及有机/无机材料的性质差异，使得自旋注人过程 难以实现, 如MSV器件制备过程中顶电极金属原子 渗入甚至短路分子层 ${ }^{[8]}$; 分子层与铁磁电极之间严 重的能级失配问题 ${ }^{[12]}$ 等. 优化器件制备工艺 ${ }^{[13,14]}$ 和 在金属/分子界面处加人缓冲层优化器件结构 ${ }^{[15 ~ 17]}$ 是 防止顶电极穿透的有效方法; 同时, 选择适当的缓冲 层还可调控铁磁电极/分子层界面的能级排布, 将有 利于自旋极化载流子的注人 ${ }^{[18]}$. 以下, 我们将从器 件制备工艺和器件结构优化两个方面总结分子自旋 器件中增强自旋注人的研究进展.

MSV中顶电极的沉积通常采用电子束蒸发、热 蒸发、磁控浌射法 ${ }^{[19]}$ 等, 这些方法会使蒸发出的金属 原子伴随巨大的热能和动能, 沉积过程中很容易渗 透到分子层中. 为削弱顶电极的动能, Sun等人 ${ }^{[13]}$ 利 用缓冲层协助生长法, 先将顶电极 Co以纳米点的形 式沉积为缓冲层, 再将剩余的顶电极继续沉积完成. 缓冲层的保护在一定程度上有效地阻止了顶电极 Co 对八羟基喹啉铝 $\left(\mathrm{Alq}_{3}\right.$ ) 的渗透(图2(a)), 但界面间仍 有微弱渗透现象发生. 随着 $\mathrm{Alq}_{3}$ 厚度的增加, 相互渗 透的厚度远远小于分子层的有效厚度, 反而表现出 高达 $300 \%$ 的GMR，表明Co对分子层渗透造成的负面 影响已经消除 ${ }^{[13]}$. Hueso研究组 ${ }^{[14]}$ 开发了一种低温速 控顶电极沉积技术, 采用液氮冷却样品基底的物理 降温方式, 有效消除顶电极金属原子的热能和动能 对分子层的破坏, 得到的MSV器件横截面的透射电 子显微镜(TEM) 图像显示器件的每层材料之间的界 限非常清晰, 无任何渗透现象(图2(b)), 表明这种低 温速控法是一种行之有效的MSV制备手段. 除了器 件制备工艺优化外, 分子层/顶电极界面处缓冲层的 构建也是消除顶电极金属材料向分子层渗透的有效 手段. 在分子自旋电子器件中, 常见的缓冲层包括 $\mathrm{Al}_{2} \mathrm{O}_{3}{ }^{[15]}, \mathrm{MgO}^{[21]}, \mathrm{LiF}^{[16,17]}$ 等. 上述MSV器件制备工 艺和结构方面的创新有效解决了顶电极渗透分子层 这一普遍存在的难题, 为分子材料中可信自旋注人 的实现提供了重要保障.

MSV中电极/分子层界面缓冲层的构建也是克服 界面能级失配问题，优化自旋注人的有效手段. 从光 电子能谱分析发现, 相比于标准 $\mathrm{Alq}_{3}$ 谱图, $\mathrm{Alq}_{3}$ 分子 与Co原子直接接触会产生 1 个额外的峰(图2(c)), 表 明两者之间存在化学作用 ${ }^{[15]}$, 因而导致自旋注人难 
(a)

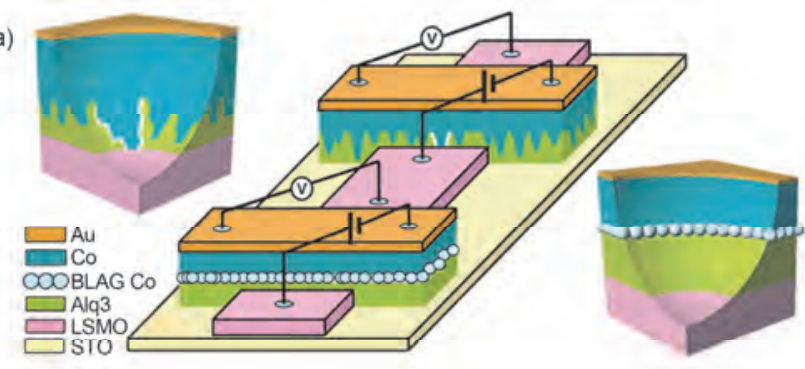

(b)

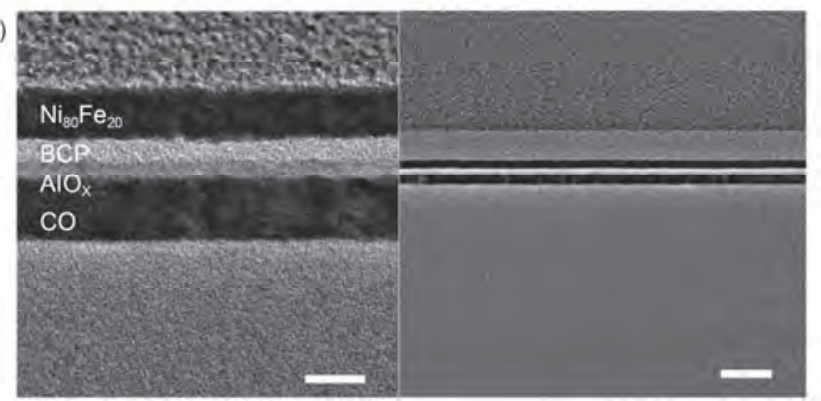

(c)
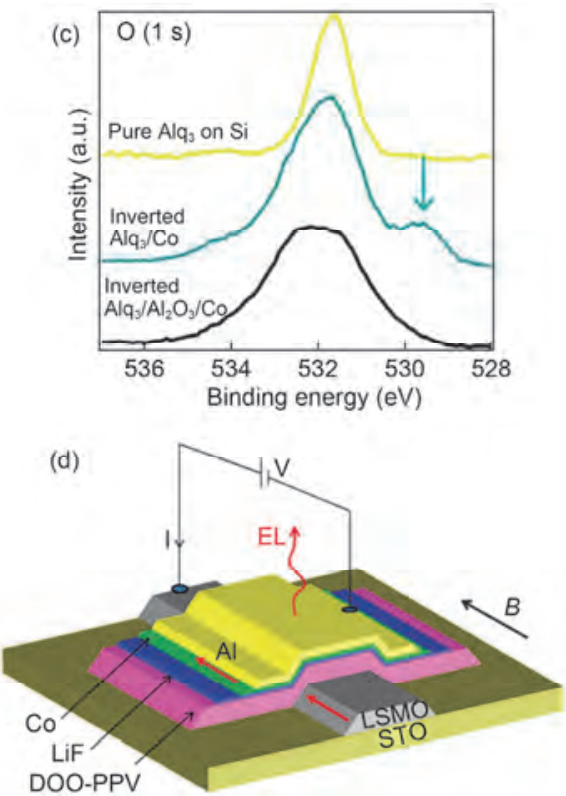

图 2 MSV自旋注人优化的手段. (a) 缓冲层协助生长法制备MSV和普通方法制备MSV对比 ${ }^{[13]}$. (b) 低温速控法制备MSV的横截面TEM图 像 ${ }^{[14]}$. (c) $\mathrm{Alq}_{3}, \mathrm{Alq}_{3} / \mathrm{Co}, \mathrm{Alq}_{3} / \mathrm{Al}_{2} \mathrm{O}_{3} / \mathrm{Co}$ 的光电子能谱 ${ }^{[15]}$. (d) 自旋发光二极管的结构示意图 ${ }^{[20]}$

Figure 2 Methods of enhancing spin injection efficiency. (a) MSVs fabricated by buffer layer assisted growth method and conventional method ${ }^{[13]}$. (b) TEM cross-sectional images of low temperature and rate-controlled $\mathrm{MSVs}^{[14]}$. (c) Photoelectron spectra of pure $\mathrm{Alq}_{3}, \mathrm{Alq}_{3} / \mathrm{Co}$, and $\mathrm{Alq}_{3} / \mathrm{Al}_{2} \mathrm{O}_{3} / \mathrm{Co}$ structure respectively ${ }^{[15]}$. (d) Schematic structure of the spin-polarized light-emitting diode ${ }^{[20]}$

以实现. 另外, Santos等人 ${ }^{[18]}$ 提出金属电极和分子层 的界面处会形成偶极层, 造成自旋注人的势垒增高. 而在金属/分子层界面处插人 1 个极薄的 $\mathrm{Al}_{2} \mathrm{O}_{3}$ 缓冲层 可以有效地消除有害界面作用 ${ }^{[15]}$, 并降低电子的注 人势垒 ${ }^{[18]}$, 实现更高效的自旋注人. 除 $\mathrm{Al}_{2} \mathrm{O}_{3}$ 外, 晶 体 $\mathrm{MgO}$ 与晶体 $\mathrm{Fe}$ 或 $\mathrm{CoFe}$ 电极相互耦合可以作为一个 近乎完美的自旋过滤层应用于分子磁隧道结中 ${ }^{[21]}$, 从而明显增强自旋注人效率.

$\mathrm{LiF}$ 作为增强自旋电子注人的界面缓冲层被广泛 应用于双极性分子自旋器件的制备中 ${ }^{[20]}$ (图 2(d)). Vardeny研究组 ${ }^{[20]}$ 关于自旋发光二极管 (spin-OLED) 的研究中, 发现具有长自旋弛豫时间的氛代聚(亚苯 基-亚乙烯基)衍生物(DOO-PPV)自旋输运层可以有 效延长注人的自旋空穴的存留时间, 但由于 Co 和 $\mathrm{La}_{0.7} \mathrm{Sr}_{0.3} \mathrm{MnO}_{3}(\mathrm{LSMO})$ 自旋极化率的差异, 导致电子 的自旋注人效率极低. 而实现高操作电压下有效的 双极化自旋注人, 进而实现有效的磁控电致发光效 应(MEL)是成功构建spin-OLED器件的重要前提. $\mathrm{LiF}$ 界面缓冲层的使用有效地提高了Co电极端的电子自 旋注人效率, 从而平衡了两端注人的自旋载流子数 量, 在分子自旋器件中测得了高达 $1 \%$ 的 MEL, 从而 成功构建了首个spin-OLED器件.

\section{3 基于分子自旋阀的自旋界面效应研究}

自旋界面是影响自旋注人效率和输运距离的关 键因素, 基于界面效应的研究是分子自旋器件性能 优化和自旋信号调控的关键方向 ${ }^{[9,22]}$. 一方面, 由于 金属和分子的相互作用导致界面处化学结构和电学 性质发生改变, 从而影响自旋载流子通过界面时的效 率; 另一方面, 插人金属和分子界面处的极性分子由 于能级排布改变或界面化学作用会使MR信号翻转.

当铁磁金属与分子层接触时, 金属表面自旋䢃 裂的 $\mathrm{d}$ 或 $\mathrm{f}$ 轨道和分子 $\pi$ 轨道发生杂化, 产生自旋䢃裂 的杂化界面态(HIS $)^{[23]}$. Fahlman研究组 ${ }^{[24]}$ 利用X射线 光电子能谱分析了 $\mathrm{Fe}$ 与 $\mathrm{Alq}_{3}$ 分子接触后产生的相互 作用, 其光谱峰值表明杂化作用发生于 Fe与其相距 较近的两个喹啉配体之间. 由X射线磁性圆二色性表 征发现, $\pi$ 共轭的 $\mathrm{Alq}_{3}$ 分子在与 $\mathrm{Fe}$ 相互作用的过程中 发生了磁化, 因而自旋信号可通过交换耦合作用实 现更加有效的自旋注人. 另外, 电子受体界面层分子 (如TNAP等)同样会由于与铁磁电极表面的相互作用 产生HIS，因此削弱空穴载流子的注人势垒进而优化 了MSV中自旋空穴的注人效率 ${ }^{[12]}$.

除此之外, HIS的电学性质会根据杂化特征和强 
度发生改变, 同时又反作用于分子层的电学结构. 在 分子层与铁磁层接触的表面, 由于较强的化学吸附, 其最高占据分子轨道(HOMO) 和最低未占据分子轨 道(LUMO)之间靠近费米能级的位置会形成占据的 HIS(oHIS)和未占据HIS(uHIS)(图3(a) $)^{[25]}$. HIS的存在 会改变铁磁层表面两种自旋状态的相对态密度 $(\mathrm{DOS})^{[23]}$. 如, 唒菁类分子 $(\mathrm{MPc})$ 与铁磁电极发生相 互作用会产生HIS, 导致自旋向上或向下的DOS分布 有不同程度的扩展. 相比之下, 第二层分子由于与铁 磁层相距较远, 两者之间只存在很弱的物理吸附作 用, 该HIS 与费米能级的能量差较大, 形成的自旋势 垒只能允许一种自旋态的载流子有效地注人, 也说 明第二层分子对自旋信号具有过滤效应 ${ }^{[26]}$. 该效应 对随后自旋载流子在整个分子层的输运十分有利. 基于第二层分子与铁磁金属界面间的自旋过滤效应, 最近, Moodera研究组 ${ }^{[27]}$ 采用一种平面的菲基分子 ZMP制备了首个基于自旋界面效应的分子自旋存储 器件(图3(b)). 在该器件中, 磁性分子与铁磁电极由 于界面自旋转移形成了一种杂化的有机金属超分子 磁性层, 因而实现了较好的自旋过滤性能和较大的 界面磁电阻. 基于界面工程的分子自旋存储器件掀
起了界面研究的热潮, 也为分子自旋器件的应用创 造一个全新的端口.

除金属和分子间产生界面效应外, MSV器件中 金属与分子层间插人极性分子界面层(如LiF)也会产 生界面效应, 从而导致MR信号的翻转, 而这一现象 的产生目前被认为与界面能级排布 ${ }^{[16]}$ 或界面化学反 应 $^{[17]}$ 有关. 从能级排布的角度分析, $\mathrm{LiF}$ 的存在会产 生界面偶极子, 从而使得真空能级下降, 而HOMO能 级也会随之下移. 自旋空穴在分子材料中主要依赖 HOMO 能级输运, 因此该能级的移动会改变另一端 铁磁电极费米能级处的DOS, 进而改变自旋极化态. 通过对比有、无LiF界面层存在的能级排布图(图3(c)), 发现同时注人的空穴载流子在另一端铁磁电极检测 到相反的自旋极化信号，从而证明是 $\mathrm{LiF}$ 界面层导致 的能级排布差异造成了MR的翻转 ${ }^{[16]}$. 另外, Hueso研 究组 ${ }^{[17]}$ 证明 $\mathrm{LiF}$ 界面层与铁磁金属电极间的化学反应 也可以导致 MR发生翻转. $\mathrm{LiF}$ 材料熔点低, 易热解离, 在热沉积过程中很容易分解产生氟离子. 氟离子与 铁磁电极发生化学反应, 生成 $\mathrm{Ni}, \mathrm{Co}$ 或 Fe的氟化物. 这些金属氟化物均为反铁磁材料, 且与底铁磁电极的 磁化方向相反, 而顶铁磁电极与反铁磁性的金属氟化 (a)

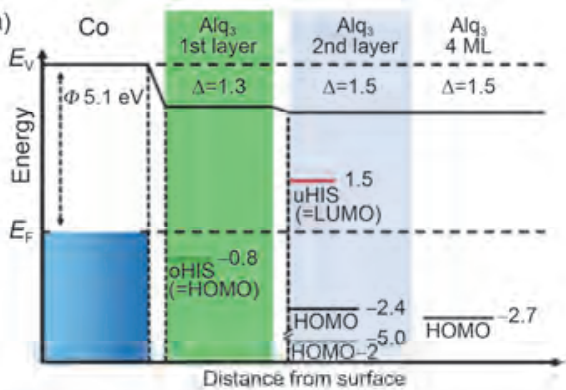

(b)

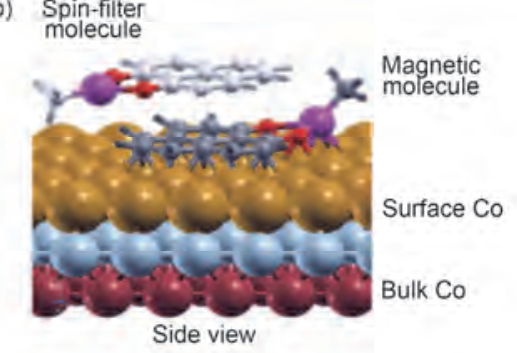

(c)

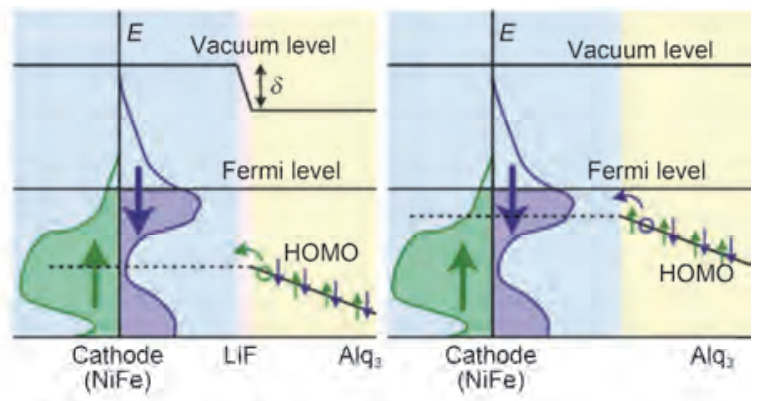

(d)

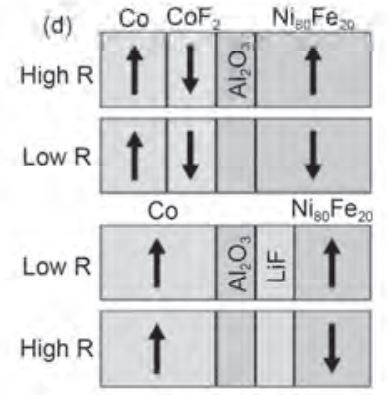

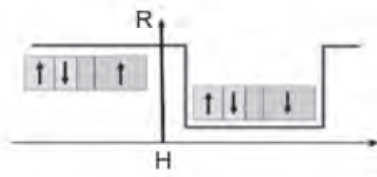

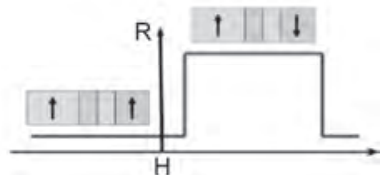

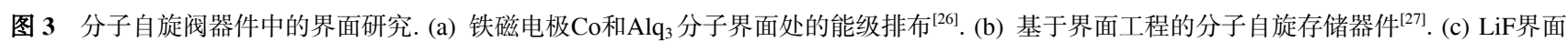
层对界面处能级排布的影响 ${ }^{[16]}$. (d) LiF的沉积顺序对MR的影响 ${ }^{[17]}$

Figure 3 Spinterfacial studies based on MSV. (a) Energy level alignment at Co/Alq ${ }_{3}$ interface ${ }^{[26]}$. (b) A molecular spin memory device based on interface engineering ${ }^{[27]}$. (c) Effect of LiF layer on energy level alignment at Metal/molecular interface ${ }^{[16]}$. (d) MR sign determined by deposited sequence of $\mathrm{LiF}^{[17]}$ 
物层的相对磁化方向决定了器件电阻的变化(图3(d)), 因而表现出完全相反的MR信号.

此外, 铁电材料, 如 $\mathrm{PbZr}_{0.2} \mathrm{Ti}_{0.8} \mathrm{O}_{3}(\mathrm{PZT})^{[28]}$, 聚 (偏二氟乙烯) $(\mathrm{PVDF})^{[29]}$ 等同样应用于 $M S V$ 的研究中, 以磁电耦合为理论基础, 在外加偏压下, 通过铁电材 料的电极化影响铁磁电极的自旋极化, 进而调控MR 的大小和翻转. 利用电场调控MR信号的研究并没有 仅仅局限于界面层的优化, 新型电存储自旋阀器件 将非易失性电存储效应与自旋阀效应结合 ${ }^{[30]}$, 同样 可以通过磁阻开关效应实现MR的大小调控. 根据预 先施加的操作偏压不同, MR调控十分明显且具有很 好的可重现性. 通过设计预施偏压, 可实现器件的多 态电阻切换, 有望突破两种电阻态的存储界限, 实现 多态信息存储 ${ }^{[31]}$.

\section{4 基于分子自旋阀的自旋输运优化及输运 机制}

自旋输运距离的提升是分子自旋电子学中最重 要的研究方向之一, 实现室温下稳定、长距离的自旋 输运是该领域长期以来存在的关键科学问题. 近年
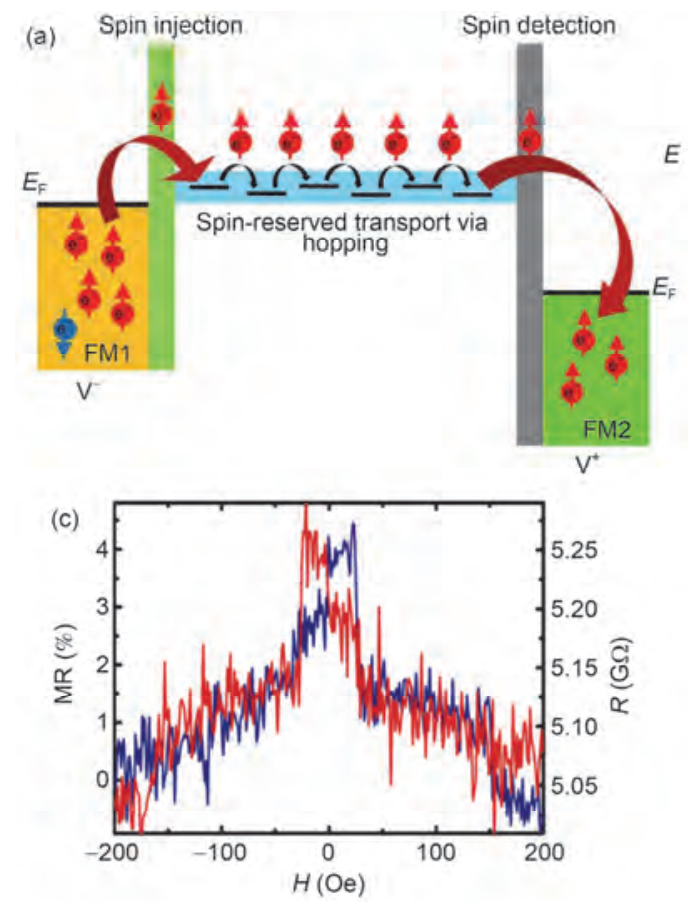

来, 分子材料的自旋输运优化和机制研究已取得了 较大的进展.

Zhang等人 ${ }^{[19]}$ 利用 $\mathrm{C}_{60}$ 分子中电子自旋弛豫时间 较长的特性, 在室温下观察到大于 $5 \%$ 的MR信号, 并 实现了高达 $110 \mathrm{~nm}$ 的室温自旋输运距离. 在该工作 中, 还阐明了自旋载流子的跃迁输运机制(图4(a)), 该输运机制与分子电子学研究中广泛验证的结论相 同, 但与此前Koopmans 研究组 ${ }^{[2,34]}$ 发现的多步隧穿 输运机制具有较大区别(图4(b)). Hueso研究组 ${ }^{[14]}$ 基于 $M S V$ 器件开展了关于跃迁和隧穿两种自旋输运机制 的研究. 研究人员通过电极与分子层之间完全氧化 的 $\mathrm{Al}_{2} \mathrm{O}_{3}$ 与半氧化的 $\mathrm{AlO}_{x}$ 界面层的构建, 分别实现了 不同模式的自旋输运，并且发现具有跃迁输运模式 的MSV器件在实现室温下长距离自旋输运方面具有 较大优势, 并基于浴铜灵(BCP)分子材料实现了超过 $60 \mathrm{~nm}$ 的室温自旋输运距离(图4(c)). 随后, Hueso课 题组 ${ }^{[35]}$ 又采用与两铁磁电极 $\left(\mathrm{Co}\right.$ 和 $\left.\mathrm{Ni}_{80} \mathrm{Fe}_{20}\right)$ 能级匹配 度更高的全氟酞菁铜 $\left(\mathrm{F}_{16} \mathrm{CuPc}\right)$ 作为自旋输运层, 通 过低温冷却方法调控形貌以得到粗鋉度更低的薄膜 表面, 制备得到的MSV器件室温自旋输运距离高

(b)

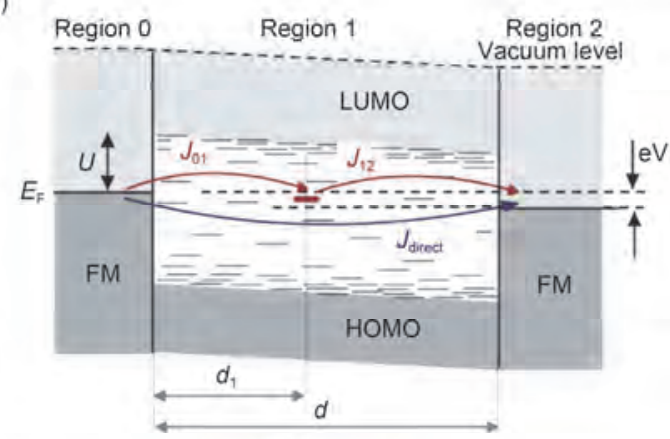

(d)

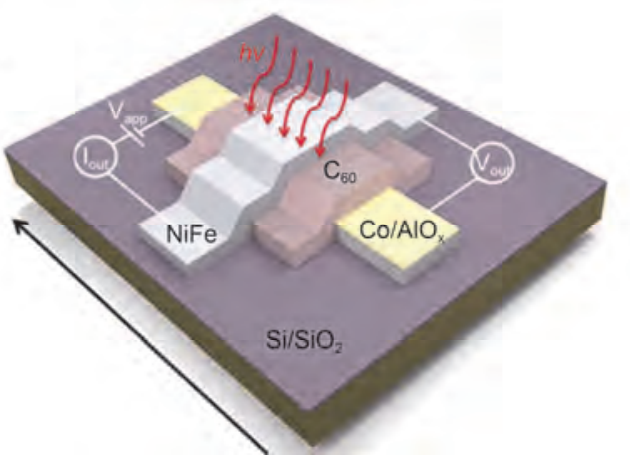

图 4 分子自旋阀中自旋输运机制和输运距离的研究. 跃迁的自旋输运机制 ${ }^{[19]}$ (a)和多步隧穿自旋输运机制 ${ }^{[32]}$ (b)示意图. (c) 基于半氧化铝的 $60 \mathrm{~nm}-\mathrm{BCP}$ 分子自旋阀的MR测试 ${ }^{[14]}$. (d) 分子自旋光伏器件结构示意图 ${ }^{[33]}$

Figure 4 Basic researches of spin transport mechanism and distance in MSV. (a) Spin transport mechanisms, hopping ${ }^{[19]}$ and (b) multistep tunneling ${ }^{[32]}$ respectively. (c) MR measurement of $60 \mathrm{~nm}-\mathrm{BCP}$ MSV with leaky-AlO ${ }_{x}^{[14]}$. (d) Schematic structure of molecular spin-photovoltaic devices ${ }^{[33]}$ 
达 $180 \mathrm{~nm}$. 除此之外, 基于逆自旋霍尔效应可检测到 通过相邻磁绝缘体中的铁磁谐振产生的纯自旋流, 且纯自旋流无能量损耗, 对实现超长距离的自旋输 运大有裨益 ${ }^{[36 ~ 39]}$.

基于自旋输运的研究基础和分子半导体材料优 异的光学性能, 首个将分子自旋阀效应与光伏效应 结合的富勒烯 $\left(\mathrm{C}_{60}\right)$ 分子自旋光伏器件(MSP)(图4(d)) 诞生, 该器件可实现自旋信号的初步操控 ${ }^{[33]}$. 在 MSP中, 光照条件下, 由于光伏效应产生的内建电场 会减弱甚至完全消除载流子的非自旋极化部分, 产 生完全极化自旋流, 因此, 在一定程度上初步实现了 对自旋信号的操控 ${ }^{[39]}$. 功能性分子自旋器件的研究 进展对分子自旋电子学走向应用具有重大意义.

\section{5 总结与展望}

分子自旋阀是分子自旋电子学的重要研究基础
和手段, 也是该研究领域中不可或缺的部分. 在过去 的十余年里, 在以提高自旋注人效率为目的的自旋 阀制备手段创新和结构优化研究方面, 自旋界面效 应以及输运距离和机制的探索方面, 都取得了非常 丰硕的研究成果. 分子自旋电子学中的器件研究作 为该领域的关键基础, 并没有局限于器件结构和性 能的优化以及相关物理机制的研究, 还包括开发可 应用于自旋器件的新型分子半导体材料 ${ }^{[40,41]}$ 及界面 材料 ${ }^{[42,43]}$ 的创新研究. 将分子半导体材料的光、电功 能性与自旋电子器件相结合, 研究分子自旋电子学 理论和器件应用, 是未来该领域的重点发展方向之 一, 在凸显分子自旋电子学研究优势的同时, 也为自 旋电子学的发展增添了色彩. 目前该研究领域已经 获得了重要进展, 但其中分子自旋器件制备、界面设 计和自旋输运等方面的研究仍然处于较为基础的阶 段, 亟待进一步的发展.

\section{参考文献}

1 Wöhrle D, Meissner D. Organic solar cells. Adv Mater, 1991, 3: 129-138

2 Kulkarni A P, Tonzola C J, Babel A, et al. Electron transport materials for organic light-emitting diodes. Chem Mater, 2004, 16: $4556-4573$

3 Horowitz G. Organic field-effect transistors. Adv Mater, 1998, 10: 365-377

4 Sanvito S. Molecular spintronics. Chem Soc Rev, 2011, 40: 3336-3355

5 Dediu V, Murgia M, Matacotta F C, et al. Room temperature spin polarization injection in organic semiconductor. Solid State Commun, 2002, 122: 181-184

6 Baibich M N, Broto J M, Fert A, et al. Giant magnetoresistance of (001)Fe/(001)Cr magnetic superlattices. Phys Rev Lett, 1988, 61: $2472-2475$

7 Žutić I. Spintronics fundamentals and applications. Rev Mod Phys, 2004, 76: 323-410

8 Xiong Z H, Wu D, Valy Vardeny Z, et al. Giant magnetoresistance in organic spin-valves. Nature, 2004, 427: 821-824

9 Cinchetti M, Alek Dediu V, Hueso L E. Activating the molecular spinterface. Nat Mater, 2017, 16: 507-515

10 Sun D L, Ehrenfreund E, Valy Vardeny Z. The first decade of organic spintronics research. Chem Commun, 2014, 50: 1781-1793

11 Alek Dediu V, Hueso L E, Bergenti I, et al. Spin routes in organic semiconductors. Nat Mater, 2009, 8: 707-716

12 Shi S W, Sun Z Y, Bedoya-Pinto A, et al. Hybrid interface states and spin polarization at ferromagnetic metal-organic heterojunctions: Interface engineering for efficient spin injection in organic spintronics. Adv Funct Mater, 2014, 24: 4812-4821

Sun X N, Gobbi M, Bedoya-Pinto A, et al. Room-temperature air-stable spin transport in bathocuproine-based spin valves. Nat Commun, 2013, 4: 2794

15 Zhan Y Q, Liu X J, Carlegrim E, et al. The role of aluminum oxide buffer layer in organic spin-valves performance. Appl Phys Lett, 2009, 94: 053301

16 Schulz L, Nuccio L, Willis M, et al. Engineering spin propagation across a hybrid organic/inorganic interface using a polar layer. Nat Mater, 2011, 10: 39-44

17 Ciudad D, Gobbi M, Kinane C J, et al. Sign control of magnetoresistance through chemically engineered interfaces. Adv Mater, 2014, 26: 7561-7567

18 Santos T S, Lee J S, Migdal P, et al. Room-temperature tunnel magnetoresistance and spin-polarized tunneling through an organic semiconductor barrier. Phys Rev Lett, 2007, 98: 016601 
Nat Commun, 2013, 4: 1392 brushes. Adv Funct Mater, 2016, 26: 3999-4006

43 Appl Phys Lett, 2009, 95: 202506 cial engineering. Adv Mater, 2017, 29: 1602739 heterojunctions. Adv Mater, 2010, 22: 1626-1630 ecules. Nat Commun, 2016, 7: 12668 509-513 4396 devices. Adv Mater, 2011, 23: 1371-1375 Phys Rev Lett, 2009, 103: 146601 single organic device. Adv Mater, 2016, 28: 2609-2615 spin-orbit coupling. Nat Mater, 2016, 15: 863-869 2010, 9: 345-352

Nguyen T D, Ehrenfreund E, Valy Vardeny Z. Spin-polarized light-emitting. Science, 2012, 337: 204-209

Szulczewski G, Tokuc H, Oguz K, et al. Magnetoresistance in magnetic tunnel junctions with an organic barrier and an $\mathrm{MgO}$ spin filter.

Jang H J, Richter C A. Organic spin-valves and beyond: Spin injection and transport in organic semiconductors and the effect of interfa-

Lach S, Altenhof A, Tarafder K, et al. Metal-organic hybrid interface states of a ferromagnet/organic semiconductor hybrid junction as basis for engineering spin injection in organic spintronics. Adv Funct Mater, 2012, 22: 989-997

Zhan Y Q, Holmström E, Lizárraga R, et al. Efficient spin injection through exchange coupling at organic semiconductor/ferromagnet

Steil S, Großmann N, Laux M, et al. Spin-dependent trapping of electrons at spinterfaces. Nat Phys, 2013, 9: 242-247

Droghetti A, Thielen P, Rungger I, et al. Dynamic spin filtering at the $\mathrm{Co} / \mathrm{Alq}_{3}$ interface mediated by weakly coupled second layer mol-

Raman K V, Kamerbeek A M, Mukherjee A, et al. Interface-engineered templates for molecular spin memory devices. Nature, 2013, 493:

Sun D L, Fang M, Xu X S, et al. Active control of magnetoresistance of organic spin valves using ferroelectricity. Nat Commun, 2014, 5:

Liang S, Yang H X, Yang H W, et al. Ferroelectric control of organic/ferromagnetic spinterface. Adv Mater, 2016, 28: 10204-10210

Hueso L E, Bergenti I, Riminucci A, et al. Multipurpose magnetic organic hybrid devices. Adv Mater, 2007, 19: 2639-2642

Prezioso M, Riminucci A, Bergenti I, et al. Electrically programmable magnetoresistance in multifunctional organic-based spin valve

Schoonus J J, Lumens P G, Wagemans W, et al. Magnetoresistance in hybrid organic spin valves at the onset of multiple-step tunneling.

Sun X N, Vélez S, Atxabal A, et al. A molecular spin-photovoltaic device. Science, 2017, 357: 677-680

Gobbi M, Golmar F, Llopis R, et al. Room-temperature spin transport in $\mathrm{C}_{60}$-based spin valves. Adv Mater, 2011, 23: 1609-1613

Sun X N, Bedoya-Pinto A, Mao Z, et al. Active morphology control for concomitant long distance spin transport and photoresponse in a

Watanabe S, Ando K, Kang K, et al. Polaron spin current transport in organic semiconductors. Nat Phys, 2014, 10: 308-313

Ando K, Watanabe S, Mooser S, et al. Solution-processed organic spin-charge converter. Nat Mater, 2013, 12: 622-627

Sun D, van Schooten K J, Kavand M, et al. Inverse spin hall effect from pulsed spin current in organic semiconductors with tunable

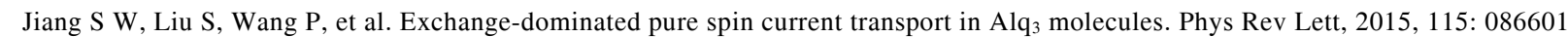

Nguyen T D, Hukic-Markosian G, Wang F, et al. Isotope effect in spin response of $\pi$-conjugated polymer films and devices. Nat Mater,

Geng R G, Roy A, Zhao W B, et al. Engineering of spin injection and spin transport in organic spin valves using $\pi$-conjugated polymer

Steim R, René Kogler F, Brabec C J. Interface materials for organic solar cells. J Mater Chem, 2010, 20: 2499-2512

3 Yip H L, Jen A K Y. Recent advances in solution-processed interfacial materials for efficient and stable polymer solar cells. Energy Environ Sci, 2012, 5: 5994-6011 
Summary for “基于分子自旋阀的自旋电子学”

\title{
Spintronic study based on molecular spin valves
}

\author{
Xianrong $\mathrm{Gu}^{1,2}$, Lidan Guo ${ }^{1,3}$, Yang Qin ${ }^{1,2} \&$ Xiangnan Sun ${ }^{1,2^{*}}$ \\ ${ }^{1}$ Key Laboratory of Nanosystem and Hierarchical Fabrication, Center for Excellence in Nanoscience of Chinese Academy of Sciences, National \\ Center for Nanoscience and Technology, Beijing 100190, China; \\ ${ }^{2}$ School of Nanoscience and Technology, University of Chinese Academy of Sciences, Beijing 100049, China; \\ ${ }^{3}$ Department of Materials Science and Engineering, College of New Energy and Materials, China University of Petroleum Beijing, Beijing 102249, \\ China \\ * Corresponding author, E-mail: sunxn@nanoctr.cn
}

Because of their extremely weak spin-orbit coupling and hence very long spin relaxation time, molecular semiconductors have considered to be very promising materials in spintronics. Since the first observation of spin transport in molecular semiconductors in 2002, molecular spintronics, as an emerging subject for studying spin-correlated phenomenon of electrons in molecular matrix, has attracted considerable research interests in the previous decade. Molecular spin valve is the most prototypical device in molecular spintronic, which should be the best test bed to study spintronic processes in molecules, including spin injection, spinterfacial effect, spin transport, as well as novel spintronic functions. Thus, to achieve highly reproducible molecular spin valves with high performances is of significant importance to the field of molecular spintronics, in which the efficient spin injection-transport process is the necessary precondition. However, according to current studies, there are several unexpected challenges for enhancing spin injection in spin valves, such as top electrode diffusion into molecular layer during device fabrication and the bad energy level alignment between ferromagnetic electrodes and molecules. To get away from these problems and improve device performance, considerable contributions have already been made to enhance spin injection from perspective of creating novel fabrication methods, optimizing device structure as well as various interfacial engineering methods. Besides, the spinterfacial effects at the interface between ferromagnetic electrodes and molecules are also considered to be crucial for spin injection. In latest studies, hybridized interface state (HIS) was proposed to characterize interaction between ferromagnetic electrode and the first molecular layer, and its spin-density of state at Fermi level of ferromagnetic electrode was changed. Therefore, spin polarization was influenced; as well its electronic structure at HIS was rebuilt to affect spin injection. In this review, research advances regarding spin transport mechanisms are also summarized, including tunneling transport and hopping transport separately. According to few recent reports, it is found that the hopping spin transport mode may avail longer spin transport distance especially at room temperature. Moreover, functional spintronic devices, integrated the spin transport and molecular functions (such as optical, electrical or spinterfacial) in one chip, are briefly introduced, which attracted considerable research interest very recently due to the great potential for applications in future. To summarize, the plentiful contributions regarding spintronic device, including performance optimization as well as mechanism study, have greatly facilitated the development of molecular spintronics in the past decade. However, molecular spintronics is an interdisciplinary filed of chemistry, physics and materials, thus exploring novel molecules that are suitable for spintronic applications must be one of the most important issues in future development of molecular spintronics.

molecular spin valve, molecular semiconductor, spin injection, spinterfacial effect, spin transport

doi: 10.1360/N972018-00836 